\title{
Accident Data For The Semantic Web
}

\author{
Ludwig Benner Jr PE (Retired) \\ Starline Software Ltd. \\ 12101 Toreador Lane \\ Oakton, VA 22124 USA
}

\begin{abstract}
This paper describes concerns about dissemination and use of lessons learned from mishap investigations, impediments posed by current practices, and opportunities for improvement. Lessons are presently developed, documented and stored primarily in narrative form and relational databases, and disseminated in many forms and media, including the Internet. Current practices pose many impediments to maximized dissemination and use. New data concepts behind the Semantic Web, exploited elsewhere, offer potential opportunities to overcome these impediments. To exploit these opportunities, formation of a working group to develop an improved Semantic Webfriendly mishap investigation lessons learning system is proposed. An example illustrating one approach to developing alternatives to the present lessons learning system is presented.
\end{abstract}

Key words: lessons learning system; investigation data structure, behavior inputoutput display.

\section{Introduction: The Need}

The need to improve adaptive dynamic behavior of socio-technical systems through investigations of accidents, before and after they happen, has long been of interest. [1] There is even a Society for Effective Lessons Learned Sharing (SELLS). [2] Maximizing development, dissemination and utilization of "lessons learned" is a continuing quest in many circles. [3-4] One US report describes the need this way:

"NASA stated that it must do a better job of communicating the various lessons learned sources to employees, improving mechanisms to link these sources, and ensuring appropriate training for employees in order to maximize lessons learning."[5]

Some organizations have established lessons learned "centers" or operating feedback systems. They make use of mishap data inputs and inputs from other sources to generate databases with lessons learned for use in those organizations [6-7] or by recognized organizations and personnel. [8-9] However the lessons learned focus on a relatively limited range of activities within those organizations.

Current investigation practices produce many kinds of outputs containing lessons learned, ranging from narrative reports, charts, completed forms, statistical trends or 
relationships, summary tables and books to bulletins, recommendation letters, check lists, training materials, or e-mail alerts. These outputs are derived by investigators or analysts who draw conclusions from the investigation or incident data.

Personal use of public lessons learned data is unknown, quantitatively, but interest in and use to generate new behaviors by individuals seems very limited. For example, one widely respected and emulated public incident lessons learned database with over 700,000 records had 88 search requests by individuals during a recent six year period.[10] How many individuals would buy a 334 page, \$US 80 book [11] to find lessons learned that might apply to their tasks and then internalize all of them to change their behaviors? How frequently do individuals change their behaviors due to desired interpretations of generalized training, procedures, standards or regulations? We don't know. However, few would argue that present practices maximize investigation lessons learned dissemination and their use by all who might benefit from the data.

These circumstances suggest that prevailing lessons learning practices for the development, communication and use of lessons learned from mishap investigations merit examination, to determine if a better lessons learning system might be developed, and how that might be accomplished.

\section{Current Learning Impediments}

What is the present system lessons learning system, and why doesn't it maximize learning from current data?

\subsection{Present Mishap Lessons Learning System.}

At present, investigators acquire, document and report "facts" or data in many forms and formats, in many diverse and often isolated systems. [12] These data are used by investigators and analysts to piece together a description and explanation of what happened, usually in narratives or on forms, using natural language. Such accident data also form the basis for conclusions about causes, cause factors, root causes, and other cause-oriented findings, from which investigators and analysts derive findings and recommendations. Findings and recommendations constitute the "lessons learned" from an investigation. Analysts then abstract, code, characterize, aggregate or otherwise refine or condense them. They are then "published" internally or made public in various kinds of media as reports, articles, papers, books, stories, graphics, training materials, check lists, etc. They also find their way into procedures or standards and regulations. The "published" data are then preserved by storage in organizational files or computerized databases for retrieval and subsequent uses at a later date.

Dissemination practices vary, but generally can be categorized as a) electronic and b) non-electronic written, verbal and graphic dissemination. [4] Electronic dissemination is achieved with computers and computerized databases, e-mails, and internet sites. Non-electronic dissemination is achieved through published or internal investigation reports, tables, checklists, on-the-job training, safety meetings, standards, training sessions, codes or regulations, and books, for example. The learning depends on the content, access to and internalization of these outputs.

Investigation data are also used for research to develop lessons learned in the form of historical trends or statistical relationships using statistical analyses or data mining 
techniques. The data are also frequently abstracted or characterized to generate lists of causes and causal factors referenced in investigation report databases, safety digests and investigation software.

\subsection{Impediments to learning.}

What are the shortcomings of the present lessons learning system?

Werner and Perry [4]cite several observed barriers to effective capture and use of investigation lessons learned. These barriers could be summarized as:

- Lessons are not routinely identified, collected and shared across organizations and industries;

- Un-organized lessons are too difficult to use, because there is too much material to search, it may be formatted differently for different reports, it's not quickly available or work pressures don't allow time or resources to find it;

- Reuse is rather ad hoc and unplanned;

- It is often hard to know what to search for or how to find useful documents; and

- Taking time to search for, identify, access and then learn from them within an organization is a problem.

Numerous observations by this author during a 35-year span disclosed additional impediments preventing maximized development and dissemination of lessons learned from investigations to all with a need to know, and their effective use of those data. These additional impediments can be characterized as:

- Current perceptions of investigation data needs that limit data presently available for sharing;

- Natural language barriers that lead to diverse source data content and structures, impeding identification of relevant behaviours;

- Data that is lost due to software obsolescence; and

- Liability concerns that motivate a desire to withhold accident data from publicly accessible sources.

Observed impediments to developing lessons learned include data gaps, logic errors, misinterpretation or misrepresentation of observations, biased data selection, flawed assumptions, and premature conclusions during investigations. All contribute to flawed development of lessons learned.

\subsubsection{Perceptions Of Data Needs}

Perceptions of what investigation data should be acquired and disseminated may be the greatest impediment to learning. Investigation purposes or mandates shape those perceptions. Investigation processes are not designed with the goal of informing all those who need to initiate new behaviors. Currently investigation inputs and outputs focus on determining the cause or cause factors, multiple causes, problems, and "root" causes, for example, from which investigators or analysts infer lessons learned to report. Outputs do not provide data in a form from which individuals can derive the specific behavioral changes they need to make. In other words, the target audience is 
spoon-fed the new behaviors deemed desirable by the "experts," in the form of recommendations.

\subsubsection{Natural Language Barriers}

The preponderance of current accident data is documented using natural language, rather than a "professional language" like those that exist in mathematics or music or medicine or other professional fields. This usage tolerates wide variations in the syntax, morphology, meaning, context and level of abstraction of documented investigation data, impeding manual analysis, machine comparisons and tabulations or rulebased manipulation like rational concatenation of elements, or interoperability, machine access and machine presentations of the data.

In these circumstances, many investigation data schemes provide accident data definition, to indicate intent and improve consistency. Data improvement efforts have typically been directed at enhancing data uniformity with guides, dictionaries or glossaries or check lists, defining words and terms. [13] However most lack a defined data structure for data that are documented. Those that do ignore the syntax and other variants, or treat them in isolation from the other impediments, without attacking the more fundamental data structure definition need. The result is that today, almost any kind of data format and structure are found in accident investigation findings and lessons learned, despite the increase in software applications [14] that require more rigor. Most lessons learned system outputs currently have low information density.

\subsubsection{Software Obsolescence.}

Some of the author's earliest digitized investigation data and records were recorded on an IBM 360 with proprietary software, and later with Wordstar and Dbase II. None are in use today. The software used to prepare those records has been made obsolete by changed hardware, operating systems and software, little of which is fully backward compatible. My point is that software obsolescence should be considered as a threat to a future lessons learning system.

\subsubsection{Liability Concerns}

Use or misuse of mishap data in litigation is a concern of many private organizations. A common reaction is to retain the data within the organization. Incident data are aggregated in voluntary reporting systems, but only when sufficiently abstracted for cause or synopsized to mask concrete identities of individual behaviors involved, as in the US Aviation Safety Reporting System (ASRS.)[15] The impeding effect of this decision on dissemination of investigation lessons learned is obvious: users with a need to know are faced with balkanized systems. Forced disclosure, through regulation or litigation, does not resolve the data needs and language issues.

\subsubsection{Other Impediments}

Other impediments I have observed that impede development, dissemination and use of lessons learned by machine include:

- Data gaps in incomplete descriptions or explanations of what happened;

- Logic errors in sequencing or coupling elements of descriptions and explanations, or in the conclusions drawn from the data; 
- Misinterpretation or misrepresentation of observations due to unsuspected biases, unwarranted assumptions, ambiguities, ambivalence or unknowns;

- Biased data selection to fit predetermined hypotheses, prior experiences, anticipated litigation posture or obstinate mind sets;

- Generalizations or abstractions masking actionable details about lessons learned;

- Premature conclusions leading to inadequately investigated or misdirected findings; and

- Rarely, deliberate falsehoods or omissions.

Present practices pose other impediments, including the inability to apply statistical analysis methods to derive findings from an episodic occurrence, and risks inherent in waiting for sufficient occurrences to discover valid statistical relationships.

\subsection{The Challenges}

The challenge is to get valid mishap-based lessons learned knowledge into the hands of the right people for their use, quickly and efficiently, to improve future performance. Ideally, lessons learned from investigations should be disseminated universally to everyone whose behavior should change to achieve safer and better task performance, so all have the opportunity to act on applicable lessons.

Each impediment poses numerous research challenges to achieving this goal; any alternative approaches need to address these challenges.

The first challenge is to define who the primary users of lessons learned data should be, and then what lessons learned data would best serve those users. Only individuals can produce new behaviors, in themselves, in objects they design or operate, or energies they manage. The investigation community needs to give priority attention to disseminating lessons learned to all individuals whose behavior could benefit from that knowledge.

A second challenge is how to overcome the natural language barriers that produce such diverse data investigation inputs and outputs, so the identified data needs can be produced and delivered to personal users in a form they can internalize directly. This will require a prescribed grammar, structure and format for investigation data from which mishap processes descriptions and explanations are developed, and from which the lessons learned are developed, The grammar, structure and format must support data sequencing, coupling and logic testing during investigations, and the machine storage, access and presentation of outputs in unambiguous behavioral terms.

A third major challenge is to define the structure and content of the lessons learning system. It must satisfy user needs, while also enabling enduring machine documentation, processing, remote access, interoperability, and utilization for timely, efficient presentation of readily internalized lessons learned behavioral information. It is unclear how the causal lens through which present practices develop investigation lessons learned could satisfy that need, suggesting the necessity for a new framework.

A fourth major challenge is the development of the system that would accommodate the transition from present practices to a newly devised lessons learning system . 
Finally, the challenges inherent in devising a comprehensive new lessons learning system such as resources, management, staffing, control, access, and ownership need to be recognized and satisfied.

\section{Potential Opportunities}

To address these challenges, any potential opportunities to improve lessons learned dissemination and use merit exploration. The exploding use of the World Wide Web to improve productivity in many fields is clearly such an opportunity waiting to be explored. Other opportunities such as previous research or developments to improve investigation processes or new investigation software may also merit consideration.

\subsection{The Semantic Web}

The Semantic Web is an evolving extension of the World Wide Web, in which web content can be expressed not only in natural language, but also in a format that can be read and used by software agents, thus permitting them to find, share and integrate information more easily. Based on progress shown, innovations related to the developments supporting the Semantic Web are creating new opportunities in many fields. [16] Developments at the World Wide Web Consortium (W3C) such as Extensible Markup Language (XML) for use on the internet are designed to describe data and focus on what data is. XML documents use self-describing and simple syntax, and are extensible: they can be extended to carry more information. XML elements can have attributes in the start tag, just like html, to provide additional information about an element. Language such as XML makes possible the introduction of self-contained, stand alone, "free-floating" data that can be utilized for analyses or displays in whatever ways are necessary to meet the user's desires. Experience in the definition and utilization of such data is already widely available, due to work in other fields by W3C working groups.

Another aspect of the opportunity offered by the Semantic Web is the ability to present text data in forms that can be readily visualized [17] increasing displayed information density of the display.

\subsection{Prior Research}

Some research has been aimed at improving investigations and the presentation of investigation data. However, lessons learned dissemination and use research is difficult to find, aside from Johnson's, ASRS's and Weaver and Perry's documents; theirs is constrained by the framework of the existing investigation world view. Research outside that world view is indicated to achieve an order of magnitude improvement in the lessons learning system. The General Systems model offers one potential alternative framework.

Experience in the definition and utilization of Web-friendly data is already widely available, due to developmental work in other fields by $\mathrm{W} 3 \mathrm{C}$ working groups. While content remains a challenge, the structural research results seem to offer a viable opportunity for progress to help develop an improved lessons learning system.

\subsection{Organizing To Address The Challenges}

Addressing these challenges and opportunities will require the capabilities of diverse experts. To organize those capabilities, the need for an Investigation Lessons Learn- 
ing System Working Group is indicated. Its task would be to develop an investigation lessons learning system that overcomes the impediments of current practices cited above, and delivers that learning data in a repeatedly accessible and readily assimilable form to all individuals who could benefit by it.

The achievements of the World Wide Web Consortium (W3C) working groups suggest a model for the organization of such a group. The mission of the W3C is to lead the World Wide Web to its full potential by developing common protocols that promote its evolution and ensure its interoperability. The $\mathrm{W} 3 \mathrm{C}$ is organized for and oversees the development of web standards. Web standards exist for programming languages, operational systems, data structures, communications protocols and electrical interfaces. The $\mathrm{W} 3 \mathrm{C}$ follows processes that promote the development of high-quality standards based on the consensus of the Membership, Team, and public. W3C processes promote fairness, responsiveness, and progress: all facets of the $\mathrm{W} 3 \mathrm{C}$ mission.

The W3C processes are described in a W3C Process Document, posted on the Internet. [18] If there is sufficient interest, the process is initiated. An initial step would be the convening of an international or intercontinental workshop or conference, to gauge the interest in the topic. After a successful workshop and discussion on an advisory mailing list, the W3C Director would announce a working group charter.

The impediments to dissemination and learning cited are offered as a possible general agenda for an initiating conference. Further analyses of dissemination and learning impediments should of course be entertained as they are identified and defined. The aim of such a conference or workshop should be at least a preliminary identification of potential data users, data needs and data structure options that a formal working group or activity might pursue. A working group should draft a list of "shall be" or "should be" mandates for investigation lessons learned data development and its structures to facilitate machine utilization.

\section{A Research Example}

An example developed during research to improve investigation processes and lessons learned creation, documentation, and dissemination shows the potential feasibility of pursuing alternative approaches.

\subsection{Data Needs.}

Prior noteworthy inquiries by Johnson [19], and others, attempting to apply rigorous logical reasoning to investigation reports, have demonstrated problems with large narrative reports and suggested remedial options. However, their focus has been primarily on the logic and presentation problems with the information in the reports, rather than lessons learned data needs, grammar or structure employed in the reports. Past and current improvements are aimed primarily at achieving data logic and consistency, to enhance relational database machine analyses of data and text mining to identify trends, identify safety improvements and prevent accidents. Our research team tried to rethink what data should be gathered, documented and made available directly to lessons learned users. Since behavioral change is the goal, it was postulated that behaviors and their relationships during the process that produced the unintended outcomes should be the research focus. 
To ensure a systematic approach, the General Systems Model was selected as the general framework, modified to reflect the behavioral focus. See Figure 1:

Figure 1 Behavioral Adaptation of General Systems Model

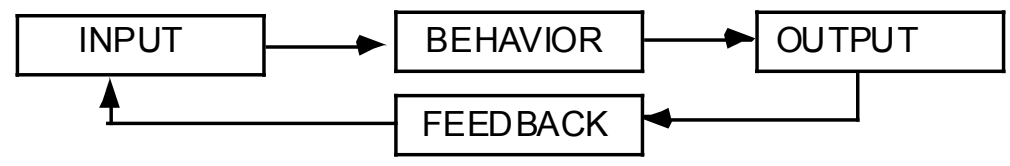

During the research it was found necessary to distinguish between data definitions offered to support current practices, and data structure definitions needed for the Semantic Web. Data Definitions in the form of natural language glossaries, dictionaries, checklists or entry instructions define the data so data providers know what data the system or forms designer wants from the provider. They are provided for use in entering data on specific forms, relational database entries or narratives. Data structure definitions on the other hand, specify the grammar, format and attributes of each stand-alone data element, with no reference to coordinate-based databases. Data designed and defined for a specific coordinate-based database has limited utility because of the ambiguity and abstractness of the natural language of investigation data .

\subsection{Data Selection}

During investigations, investigators create "building blocks" which they use to construct a description of what happened and explanation of why it happened. Many kinds of building blocks exist for this purpose, including building blocks created for investigation software. The most fully formalized behavioral investigation building blocks available, originally developed for manual implementation as a product of prior research in 1976 and refined through subsequent use, were selected for the research. See Figure 2. [20] They met the behavior data needs, and had the further advantage of having well defined data elements, grammar and structure.

\subsubsection{Building Block Example}

The building blocks were originally conceived and created to define for investigators the format and grammar for documenting observations during investigations. By transforming investigator's observations into this actor + action-based building block format, the behaviors can be properly described, ordered, linked, tested and utilized to show the logical flow of the interactions needed to produce the outcomes of interest.

Figure 2 Investigation Data Building Blocks

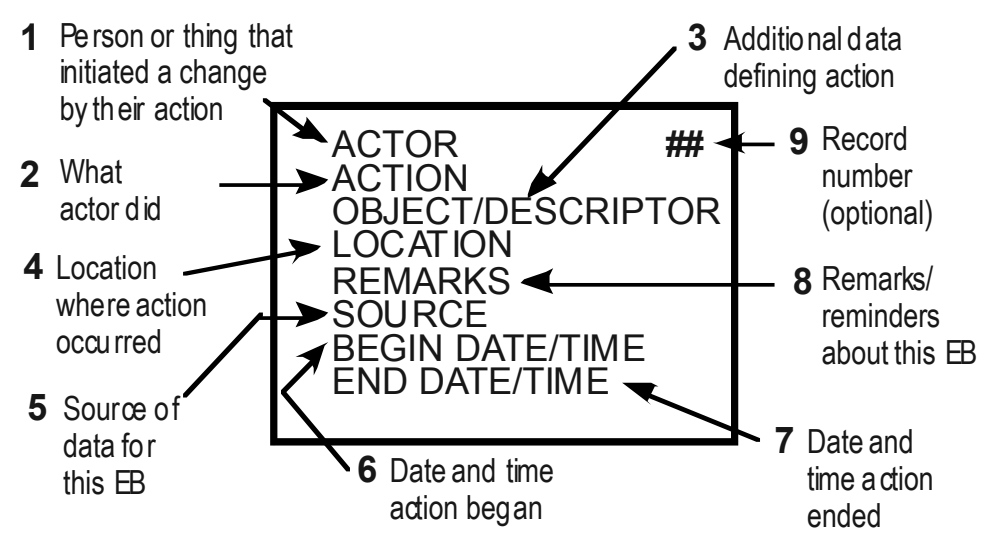




\subsubsection{XML Investigation Building Blocks}

To adapt these building block elements to the Web, these manual investigation building block data elements were configured in an XML document structure. XML was chosen over a coordinate-based SQL database, because it also offered relatively easy reliable data entry and consistency; file content flexibility; and investigation data entry editing, access, search, parsing, linking, integration and display ease. The XML document structure that resulted is shown in Figure 3.

Figure 3. Investigation Building Block Elements in XML Document *

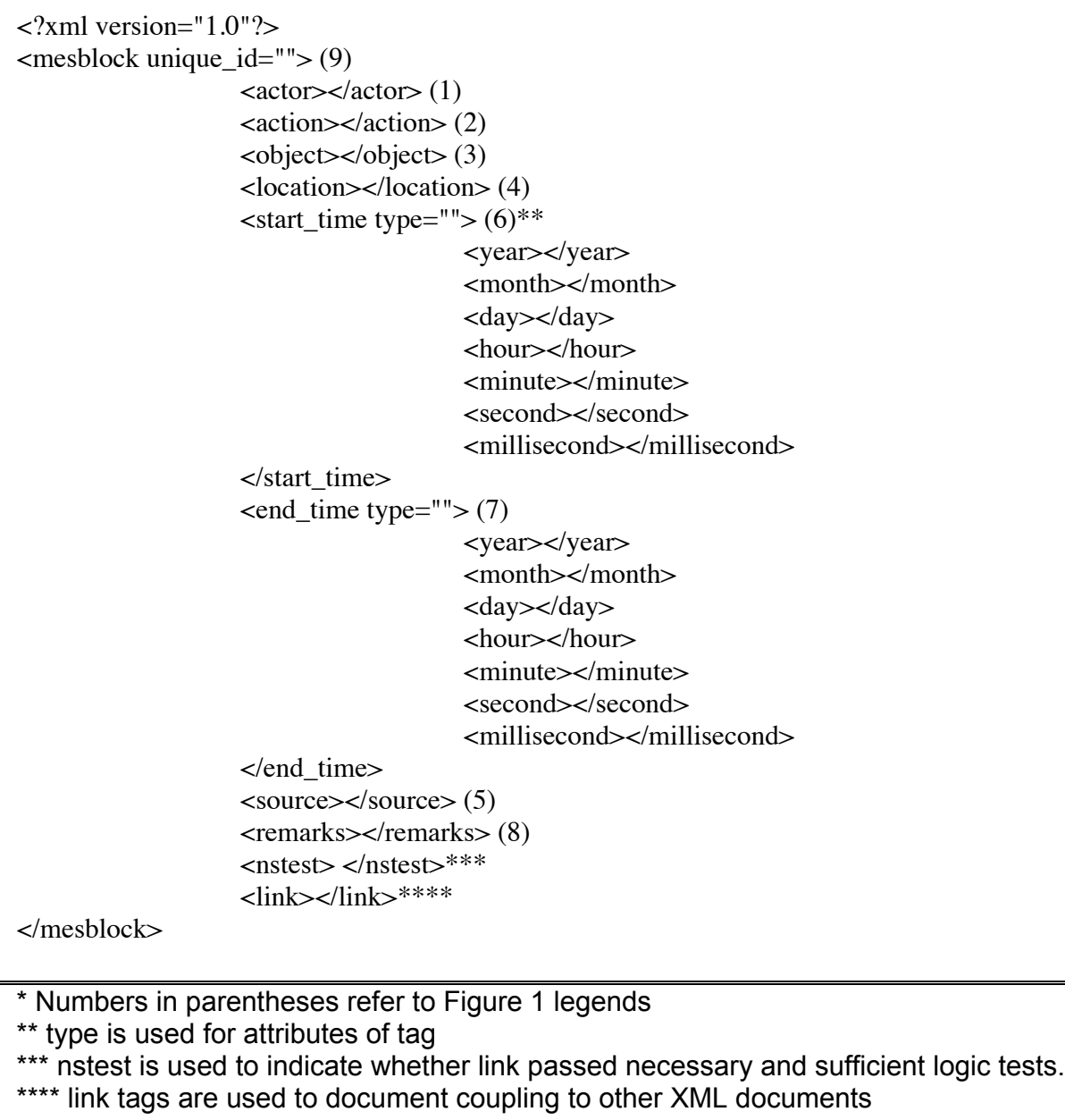

${ }^{*}$ Numbers in parentheses refer to Figure 1 legends

** type is used for attributes of tag

${ }^{* * *}$ nstest is used to indicate whether link passed necessary and sufficient logic tests.

**** link tags are used to document coupling to other XML documents

Like other files created for display on the internet, the structure uses tags for data elements and other purposes. The first line of the document is the XML declaration. The "mesblock" tag is the XML root element, which is given a unique identification attribute to distinguish the document from all others. The other tags are XML document elements or building block element tags, having attributes consisting of the data.

\subsubsection{Expanding The Data}

The tags with number suffixes were the manual building block elements added to the XML document first. The remaining tags were added to meet additional needs as the document was used to create experimental graphic and tabular outputs. For example, sometimes events were separated by milliseconds, so provision had to be made for more detailed time data. Then it was found necessary to add new XML elements to 
accommodate the links and linked events $(<\operatorname{link}></$ link $>)$, and the logic test status indicator element $(<$ nstest $></$ nstest $>)$ to indicate the state of completion.

Later, the scope of the research was expanded beyond investigation to include problem definition and recommendation development. This necessitated linking a separate file to the building block file, to accommodate conventional problem statement displays, statement analyses, problem solving options, their analysis and assessment, and recommendation selection findings.

Note that conditions are not included as elements; all the elements and attributes refer to behavior events. The rationale for excluding conditions was that conditions remain unchanged until someone or something acts on a condition to change it. Thus the focus led to behaviors in the form of actions that changed conditions.

\subsection{Building Block Uses}

XML event building block (EB) files with data entry and editing software were developed. Then software to machine read the XML files was developed to generate several kinds of graphic event flow charts, glossaries, input-output links among two or more coupled EBs, jump maps, sortable tabular EB displays, and parsed text files. Display data were machine converted to web-compatible graphic files that could be processed for distribution on the internet. Examples of hard copies or Internet files of the outputs mentioned can be provided upon request to the author.

Web pages, which provided for the remote entry and capture of XML building block data with any $\mathrm{W} 3 \mathrm{C}$ compatible computer browser were also created. These data files have been stored on web sites for participating investigators to edit and use. Data files were concatenated, printed or saved and stored as graphics files for dissemination on the internet as they were created, to show all investigators on the case the status of an investigation in real time. At the end of an investigation, the completed data would be presented graphically, in tabular form or as text phrases for inclusion in reports.

The ability to easily concatenate XML-based EB files permitted concurrent conduct of investigation tasks and individual file preparation duties by two or more investigators. Their data files can be combined into one project file as new contributions become available. When more investigations were documented, aggregated data files for groups of investigations were created. The aggregated files enabled tabular listings of all event building blocks, which can be screened to find common event building blocks across all the incidents in the new file, with detailed information about each building block's inputs and outputs in the file..

\subsection{Event Set Displays}

For lessons learning purposes, one of the most useful outputs of this research was the process of developing "event set" displays of coupled building blocks. All the behavior inputs necessary to produce a mishap outcome could be displayed in a systems-based tabular form, in the sequence they occurred. This searchable display provided every input to each behavior disclosed by the investigation, and also every output that each behavior produced. It has been termed our "Event Block Input/Output (EBIO)" array, as shown in Figure 4. 
Figure 4. EBIO Array of Event Sets

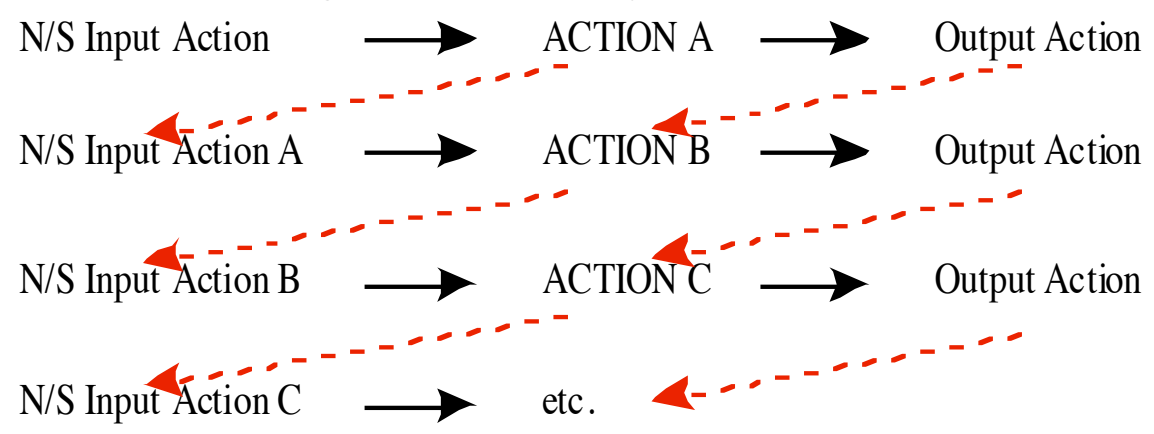

Figure 5 is a clipping from a work file for analysing an accident investigation report published in an investigation guide, illustrating the EBIO structure.

Figure 5. Example of EBIO For Selected Event Sets

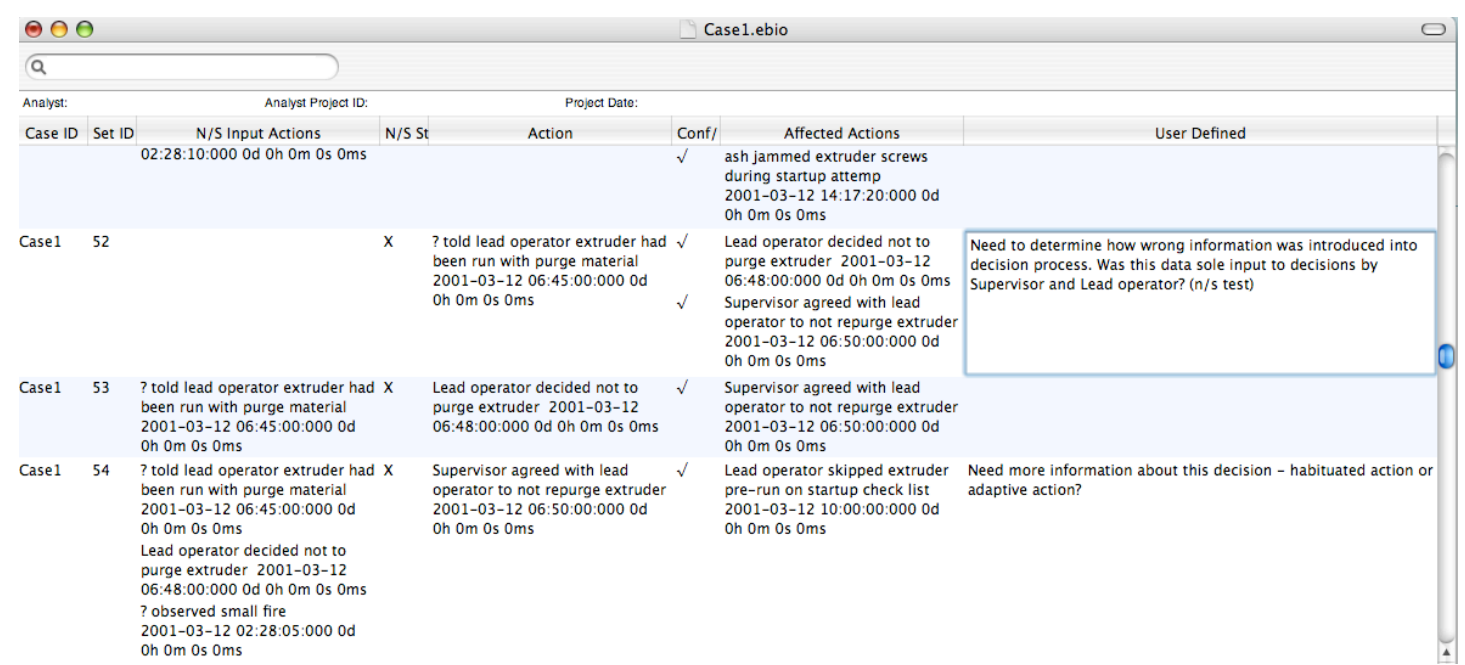

This is a work in process. Note the gaps in the flow of inputs and outputs which need to be resolved before the investigation is closed, as indicated by the investigator's comments. When the gaps are closed, the input-output flow should define how those behaviors advanced the mishap process. Viewers should be able to associate these behaviors with their own if they are relevant to their workplace.

Note also that "cause" plays no role. Unambiguous input behaviors and output behaviors in the form of specific actors/actions do.

When posted on the web, individuals can search EBIO files concatenated from many cases for event sets involving their tasks, and see behavior sets to avoid or modify. This direct association and recurrent accessibility should facilitate internalization of the lessons displayed and their use. This research is ongoing.

\subsubsection{Conducting Investigations.}

The graphic, tabular and EBIO displays created from the XML building block files help investigators during investigations, by displaying the flow of coupled events created from the investigation data already acquired. Any gaps in the flow of the events indicate a need for better understanding (e.g., more data) to complete the investiga- 
tion. It offers guidance for interviewers by showing what an actor did during the accident process, with gaps indicating behaviors not yet identified, as in Figure 5.

\subsubsection{Disseminating Lessons Learned.}

This EBIO display provided a way to disseminate investigation "lessons learned" data, although in an unconventional, high information intensity format. Each input/behavior/output event set offers a concrete description of part of a mishap process, which if replicated can play a role in another accident or near miss; no expert interpretations are needed. Concatenated case files can identify event set patterns within or across activities. Access to such data should help all with a need to know, including writers of specifications, procedures, standards, regulations, guides and training materials, for example. Repeated access should facilitate the behavior change process.

\subsubsection{Minimizing ambiguities.}

Ambiguities in actor names are easily avoided by naming each actor uniquely. Avoiding ambiguities in documenting the actions is a greater challenge, because of natural language ambiguities in most languages. Consistent verb tense and active voice help, but diligence is required to ensure unambiguous concrete action words in the behavior descriptions.

\section{Conclusions.}

Current impediments to maximizing development, dissemination and use of lessons learned posed by present investigation practices call for change to ensure the timely development of, access to and efficient delivery of needed lessons learned information to all who should know about it and internalize it. This requires a focus on needs of users and collaborative efforts from individuals with a wide variety of expertise, for which an Investigation Lessons Learning System Working Group is proposed.

By refocusing on investigation data needed by users of lessons learned and behavior inputs and outputs, an example of a systems-based alternative to present practices was identified. It enables investigators to develop and users to access task-related lessons learned in a new, direct and readily assimilable way without expert analysts' intervention. This supports a reasonable expectation that an alternative Semantic Web-friendly lessons learning system that overcomes the impediments posed by the present lessons learning system could be developed.

\section{Acknowledgments}

The contributions of William D. Carey to the research concepts and this paper, and Tobias Haughton to the software development research, are gratefully acknowledged.

\section{References}

1. Johnson, C.W., (1999) "Improving the Presentation of Accident Reports over the World Wide Web" Proceedings of the 17th International Systems Safety Conference, The Systems Safety Society, Unionville, Virginia, USA, p 396-405, (http://www.dcs.gla.ac.uk/ johnson/papers/Web accidents/paper.html)

2. (2003) http://www.hss.doe.gov/csa/analysis/ll/sells/faqs/LLinfo.pdf

3. See Model Minimum Uniform Crash Criteria (MMUCC) project (2003). The purpose of MMUCC is to provide a minimum, standardized data set for describ- 
ing crashes of motor vehicles that will generate the information necessary to improve highway safety in each state and nationally.

(http://www.mmucc.us/index.htm)

4. Werner, P. and Perry, R., (2004) The Role of Lessons Learned in The Investigate, Communicate, Educate Cycle for Commercial Aviation, System Safety Society Proceedings of the 35th Annual International Gold Coast, Queensland, Australia, August 30-September 2, 2004, pages 51-56.

5. United States General Accounting Office (2002) Report to the Subcommittee on Space and Aeronautics, Committee on Science, House of Representatives, NASA: Better Mechanisms Needed for Sharing Lessons Learned, GAO-02195 ,

6. See a listing of Lessons Learned Centers at http://call.army.mil/links/lessons.asp

7. Dien, Y and Llroy, M., (2004) Effects of the Columbia Space Shuttle Accident on High-Risk Industries, or Can we Learn Lessons From Other Industries, presented to "HAZARDS XVIII-PROCESS SAFETY-SHARING BEST PRACTICE” 23-25 November 2004, Weston Building, UMIST, Manchester

8. For an example, see http://www.wildfirelessons.net/Home.aspx (2007)

9. European Commission Major Accident Reporting System (MARS), serving member states.

10. ASRS Program Review, Database Search Requests, Search Inquiries by Organization (2005) http://asrs.arc.nasa.gov/briefing/br 26.htm

11. Kletz, T.A., (2001) Learning from Accidents, Third Edition, Gulf Professional Publishing, Oxford England

12. Sklet, S. (2003) Comparison of Some Selected Methods for Accident Investigation, JRC - ESReDA Seminar on SAFETY INVESTIGATION OF ACCIDENTS, EC - DG JRC, Petten, Netherlands 12 May 2003

13. For example see the European Community Road Accident Database Glossary http://ec.europa.eu/transport/roadsafety library/care/doc/care glossary.pdf

14. See http://www.iprr.org/tools/softw.html for a list of software reviewed (2007)

15. ASRS Database Online (2007) with reporting and search capabilities accepts .cvs or .xls formatted spreadsheet or tabular input data http://akama.arc.nasa.gov/ASRSDBOnline/QueryWizard Begin.aspx

16. For an extensive list of examples of applications in Europe, see XML Applications at Work (2007) at http://www.softwareag.com/xml/dt/default.htm

17. Neumann, E. K., (2007) Visualizing the Semantic Web, Bio-IT World.com, July-August 2007 "When working with data, it is just as important to visualize it properly as it is to process it."

18. W3C Process Document describing Working Group operation is posted at http://www.w3.org/2005/10/Process-20051014/intro.html

19. Johnson, C.W. (1999) Improving the Presentation of Accident Reports on the World Wide Web, which argues that image maps, VRML models and QuicktimeVR techniques might be used to improve both the quality and structure of these electronic documents posted by investigation agencies. Emphasis was on documents, not lessons learned. (http://www.dcs.gla.ac.uk/ johnson/papers/Web accidents/paper.html)

20. Benner, L. (2002) 10 MES Investigation Guides, Starline Software Ltd, Oakton, VA 22124 http://www.starlinesw.com/product/Guides/MESGuide00.html 